Saudi Journal of Medicine

Abbreviated Key Title: Saudi J Med ISSN 2518-3389 (Print) |ISSN 2518-3397 (Online) Scholars Middle East Publishers, Dubai, United Arab Emirates Journal homepage: https://saudijournals.com/sjm

Original Research Article

\title{
A Study of Clinical and Laboratory Screening of Thyroid Gland
}

\author{
Dr. Praveena Kumara*
}

Associate Professor, Department of General Medicine, Kanachur Institute of Medical Sciences, Mangalore-Thokkottu-Konaje University Rd, Kotekar Village, Deralakatte, Karnataka 575018, India

DOI: $10.36348 /$ sjm.2020.v05i04.006 | Received: 31.03 .2020 | Accepted: 08.04.2020 | Published: 13.04 .2020

*Corresponding author: Dr. Praveena Kumara

\section{Abstract}

The disease of the thyroid gland is very common in our country. Though the disease is very common in our country it is often misdiagnosed or neglected thus resulting in a catastrophe for the patient. This study puts in an effort to understand the role of USG in screening the Thyroid Gland. The problem is the abnormalities can be recorded more in the youths which is directly responsible for the health care and economic burden.

Keywords: USG, Thyroid Gland, Benign, Malignant, Atypical.

Copyright @ 2020: This is an open-access article distributed under the terms of the Creative Commons Attribution license which permits unrestricted use, distribution, and reproduction in any medium for non-commercial use (NonCommercial, or CC-BY-NC) provided the original author and source are credited.

\section{INTRODUCTION}

The disease of the thyroid gland is very common in our country. Though the disease is very common in our country it is often misdiagnosed or neglected thus resulting in a catastrophe for the patient. This study puts in an effort to understand the role of USG in screening the Thyroid Gland. The problem is the abnormalities can be recorded more in the youths which is directly responsible for the health care and economic burden. Thyroid pathologies is often found in our country. Thyroid Gland is one of the most important endocrine gland in the human body as it regulates a number of metabolic functions. The disease of the thyroid gland is very common in our country [1$3]$. Though the disease is very common in our country it is often misdiagnosed or neglected thus resulting in a catastrophe for the patient. According to studies one in every ten adults in India is suffering from the thyroid disorder [4, 5]. Hypothyroidism is more commonly encountered than the hyperthyroidism. If undetected and untreated for a long time then it results in a plethora of other metabolic disorders. Women are more affected than men and this may be due to the fact that it is the only endocrine organ which directly depends on the iodine from the external source for producing the hormones [6, 7]. Early diagnosis and prompt treatment is needed so USG is such one screening option. Earlier radioactive materials were tagged and then injected to the body for diagnosing but now USG has slowly taken over and is widely used in the screening procedures of the thyroid diseases. This study puts in an effort to understand the role of USG in screening the Thyroid Gland.

Aims and Objectives: To study the role of USG in screening the Thyroid Gland.

\section{MATERIALS AND METHODS}

- This study was done in A.J Institute of Medical Sciences, Mangalore. The study was done from April 2015 to Mar 2016.

- The study was done in the Department of Internal Medicine and Sixty patients were selected for the same.

\section{Inclusion Criteria}

- Patients with thyroid diseases

- Age of the patient above 20 years. This was done to eliminate the age-related bias.

\section{Exclusion Criteria}

- Patients who were on medication for thyroid disease

- Patients who was on chemotherapy for already known malignant diseases of the thyroid.

\section{RESULTS}

Table-1: Mean age

\begin{tabular}{|l|l|l|}
\hline Mean Age & Range & Standard Deviation \\
\hline 29.82 years & $20-60$ years & 17.71 years \\
\hline
\end{tabular}




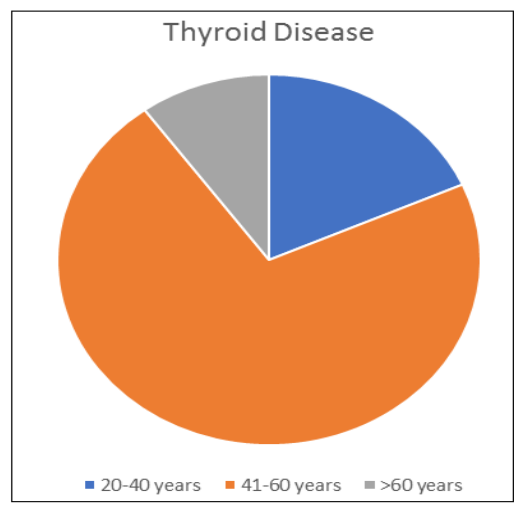

Table-2: Clinical signs and symptoms

Fig-1: Age Distribution

\begin{tabular}{|l|l|}
\hline Fever & 21 \\
\hline Pain & 17 \\
\hline Myalgia & 51 \\
\hline Dizziness & 29 \\
\hline Menorrhagia & 31 \\
\hline Tiredness & 54 \\
\hline Weight gain/weight loss & 57 \\
\hline Brady/Tachy cardia & 60 \\
\hline Blood pressure changes & 39 \\
\hline perspiration & 38 \\
\hline
\end{tabular}

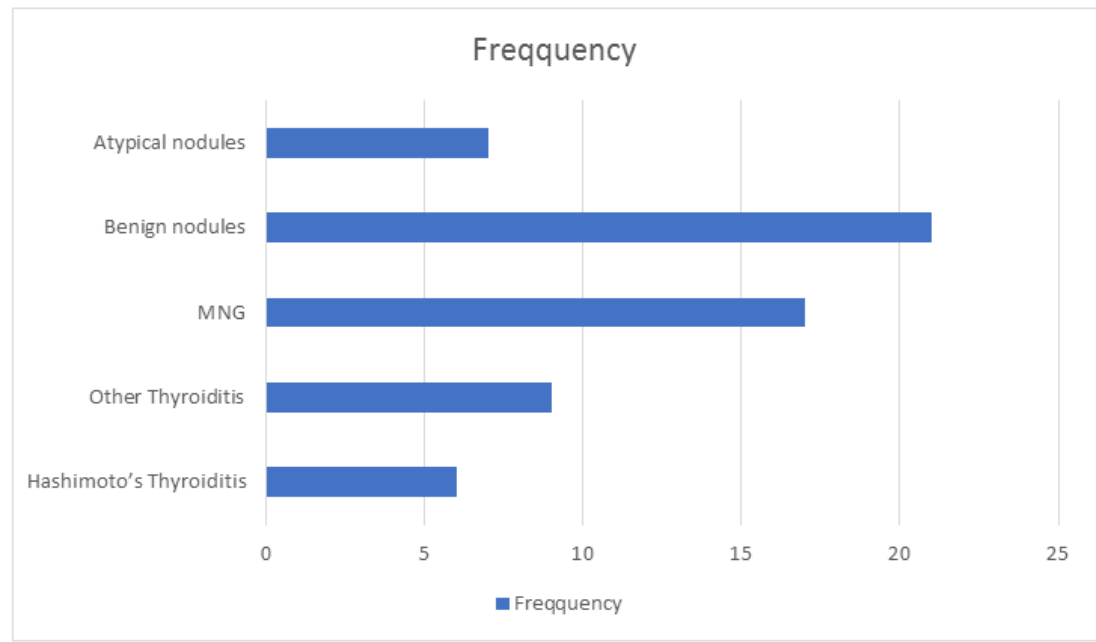

Fig-2: Thyroid Pathologies according to laboratories:

\section{DISCUSSION}

Excessive production of the thyroid hormones is called hyperthyroidism. Causes include Graves disease, toxic multinodular goitre, solitary thyroid adenoma, inflammation, and a pituitary adenoma which secretes excess TSH. Another cause is excess iodine availability, either from excess ingestion, induced by the drug amiodarone, or following iodinated contrast imaging.

Hyperthyroidism often causes a variety of nonspecific symptoms including weight loss, increased appetite, insomnia, decreased tolerance of heat, tremor, palpitations, anxiety and nervousness. In some cases it can cause chest pain, diarrhoea, hair loss and muscle weakness. Such symptoms may be managed temporarily with drugs such as beta blockers.

Long-term management of hyperthyroidism may include drugs that suppress thyroid function such as propylthiouracil, carbimazole and methimazole. Alte rnatively, can be used to destroy thyroid tissue: radioactive iodine is selectively taken up by thyroid cells, which over time destroys them. The chosen firstline treatment will depend on the individual and on the country where being treated. Surgery to remove the thyroid can sometimes be performed as a transoral thyroidectomy, a minimally-invasive procedure. Surgery does however carry a risk of damage to the parathyroid glands and the recurrent laryngeal nerve, which innervates the vocal cords. If the entire thyroid gland is removed, hypothyroidism will inevitably result, and thyroid hormone substitutes will be needed.

An underactive thyroid gland results in hypothyroidism. Typical symptoms are abnormal weight gain, tiredness, constipation, heavy menstrual bleeding, hair loss, cold intolerance, and a slow heart rate. Iodine deficiency is the most common cause of hypothyroidism worldwide, and the autoimmune disease Hashimoto's thyroiditis is the most common cause in the developed world. Other causes include congenital abnormalities, diseases causing transient inflammation, surgical removal or radioablation of the thyroid, the drugs amiodarone and lithium, amyloidosis, and sarcoidosis. Some forms of hypothyroidism can result in myxedema and severe cases can result in myxedema coma.

Hypothyroidism is managed with replacement of the hormone thyroxine. This is usually given daily as an oral supplement, and may take a few weeks to become effective. Some causes of hypothyroidism, such as Postpartum thyroiditis and Subacute thyroiditis may be transient and pass over time, and other causes such 
as iodine deficiency may be able to be rectified with dietary supplementation.

Graves' disease is an autoimmune disorder that is the most common cause of hyperthyroidism. In Graves' disease, for an unknown reason autoantibodies develop against the thyroid stimulating hormone receptor. These antibodies activate the receptor, leading to development of a goitre and symptoms of hyperthyroidism, such as heat intolerance, weight loss, diarrhoea and palpitations. Occasionally such antibodies block but do not activate the receptor, leading to symptoms associated with hypothyroidism. In addition, gradual protrusion of the eyes may occur, called Graves' ophthalmopathy, as may swelling of the front of the shins. Graves' disease can be diagnosed by the presence of pathomnomonic features such as involvement of the eyes and shins, or isolation of autoantibodies, or by results of a radiolabelled uptake scan. Graves' disease is treated with anti-thyroid drugs such as propylthiouracil, which decrease the production of thyroid hormones, but hold a high rate of relapse. If there is no involvement of the eyes, then use of radioactive isotopes to ablate the gland may be considered. Surgical removal of the gland with subsequent thyroid hormone replacement may be considered, however this will not control symptoms associated with the eye or skin.

Thyroid nodules are often found on the gland, with a prevalence of 4-7\%.The majority of nodules do not cause any symptoms, thyroid hormone secretion is normal, and they are non-cancerous. Non-cancerous cases include simple cysts, colloid nodules, and thyroid adenomas. Malignant nodules, which only occur in about $5 \%$ of nodules, include follicular, papillary, medullary carcinomas and metastases from other sites Nodules are more likely in females, those who are exposed to radiation, and in those who are iodine deficient.

When a nodule is present, thyroid function tests determine whether the nodule is secreting excess thyroid hormones, causing hyperthyroidism. When the thyroid function tests are normal, an ultrasound is often used to investigate the nodule, and provide information such as whether the nodule is fluid-filled or a solid mass, and whether the appearance is suggestive of a benign or malignant cancer. A needle aspiration biopsy may then be performed, and the sample undergoes cytology, in which the appearance of cells is viewed to determine whether they resemble normal or cancerous cells.

The presence of multiple nodules is called a multinodular goitre; and if it is associated with hyperthyroidism, it is called a toxic multinodular goitre.

An enlarged thyroid gland is called a goitre. Goitres are present in some form in about 5\% of people, and are the result of a large number of causes, including iodine deficiency, autoimmune disease (both Graves' disease and Hashimoto's thyroiditis), infection, inflammation, and infiltrative disease such as sarcoidosis and amyloidosis. Sometimes no cause can be found, a state called "simple goitre".

Some forms of goitre are associated with pain, whereas many do not cause any symptoms. Enlarged goitres may extend beyond the normal position of the thyroid gland to below the sternum, around the airway or esophagus. Goitres may be associated with hyperthyoidism or hypothyroidism, relating to the underlying cause of the goitre. Thyroid function tests may be done to investigate the cause and effects of the goitre. The underlying cause of the goitre may be treated, however many goitres with no associated symptoms are simply monitored.

Inflammation of the thyroid is called thyroiditis, and may cause symptoms of hyperthyroidism or hypothyroidism. Two types of thyroiditis initially present with hyperthyroidism and are sometimes followed by a period of hypothyroidism - Hashimoto's thyroiditis and postpartum thyroiditis. There are other disorders that cause inflammation of the thyroid, and these include subacute thyroiditis, acute thyroiditis, silent thyroiditis, Riedel's thyroiditis and traumatic injury, including palpation thyroiditis.

Hashimoto's thyroiditis is an autoimmune disorder in which the thyroid gland is infiltrated by the lymphocytes B-cell and T-cells. These progressively destroy the thyroid gland. In this way, Hasimoto's thyroiditis may have occurred insidiously, and only be noticed when thyroid hormone production decreases, causing symptoms of hypothyroidism. Hashimoto's is more common in females than males, much more common after the age of 60 , and has known genetic risk factors. Also more common in individuals with Hashimoto's thyroiditis are type 1 diabetes, pernicious anaemia, Addison's disease vitiligo.

Postpartum thyroiditis occurs in some females following childbirth. After delivery, the gland becomes inflamed and the condition initially presents with a period of hyperthyroidism followed by hypothyroidism and, usually, a return to normal function. The course of the illness takes place over several months, and is characterised by a painless goitre. Antibodies against thyroid peroxidase can be found on testing. The inflammation usually resolves without treatment, although thyroid hormone replacement may be needed during the period of hypothyroidism.

The most common neoplasm affecting the thyroid gland is a benign adenoma, usually presenting as a painless mass in the neck. Malignant thyroid cancers are most often carcinomas, although cancer can occur in any tissue that the thyroid consists of, 
including cancer of C-cells and lymphomas. Cancers from other sites also rarely lodge in the thyroid. Radiation of the head and neck presents a risk factor for thyroid cancer, and cancer is more common in women than men, occurring at a rate of about 2:1.

In most cases, thyroid cancer presents as a painless mass in the neck. It is very unusual for thyroid cancers to present with other symptoms, although in some cases cancer may cause hyperthyroidism. Most malignant thyroid cancers are papillary, followed by follicular, medullary, and thyroid lymphoma. Because of the prominence of the thyroid gland, cancer is often detected earlier in the course of disease as the cause of a nodule, which may undergo fine needle aspiration. Thyroid function tests will help reveal whether the nodule produces excess thyroid hormones. A radioactive iodine uptake test can help reveal the activity and location of the cancer and metastases.

Thyroid cancers are treated by removing the whole or part of thyroid gland. Radioactive may be given to radioablate the thyroid. Thyroxine is given to replace the hormones lost and to suppress TSH production, as TSH may stimulate recurrence. With the exception of the rare anaplastic thyroid cancer, which carries a very poor prognosis, most thyroid cancers carry an excellent prognosis and can even be considered curable.

Iodine deficiency, most common in inland and mountainous areas, can predispose to goitre - if widespread, known as endemic goitre [1]. Pregnant women deficient of iodine can give birth to infants with thyroid hormone deficiency [2,3]. The use of iodised salt to add iodine to the diet [4] has eliminated endemic cretinism in most developed countries [5], and over 120 countries have made the iodination of salt mandatory $[6,7]$.

Because the thyroid concentrates iodine, it also concentrates the various radioactive isotopes of iodine produced by nuclear fission. In the event of large accidental releases of such material into the environment, the uptake of radioactive iodine isotopes by the thyroid can, in theory, be blocked by saturating the uptake mechanism with a large surplus of nonradioactive iodine, taken in the form of potassium iodide tablets. One consequence of the Chernobyl disaster was an increase in thyroid cancers in children in the years following the accident [8].
Excessive iodine intake is uncommon and usually has no effect on the thyroid function. Sometimes though it may cause hyperthyroidism, and sometimes hypothyroidism with a resulting goitre [9].

\section{CONCLUSION}

USG is a non - invasive and non - radiational screening method. It is slowly becoming the gold standard to method of screening for thyroid pathologies.

\section{REFERENCES}

1. Unnikrishnan, A. G., Kalra, S., Sahay, R. K., Bantwal, G., John, M., \& Tewari, N. (2013). Prevalence of hypothyroidism in adults: An epidemiological study in eight cities of India. Indian journal of endocrinology and metabolism, 17(4), 647-652.

2. Sushmi, D. (2015). 1 in 10 Indians suffer from thyroid disorder: study. The times of India. Disorder Study/articleshow/46007453.cms

3. Unnikrishnan, A. G., \& Menon, U. V. (2011). Thyroid disorders in India: an epidemiological perspective. Indian Journal Endocrinol Metab, 15(Supp12):78-81.

4. Nagarkar, R., Roy, S., Akheel, M., Palwe, V., Kulkarni, N., \& Pandit, P. (2015). Incidence of thyroid disorders in India: An institutional retrospective analysis. International Journal of Dental and Medical Specialty, 2(2), 19-23.

5. Nimmy, N. J., Aneesh, P. M., Narmadha, M. P., Udupi, R. H., \& Binu, K. M. (2012). A survey on the prevalence of thyroid disorder induced by demography and food habits in South Indian population. Indian Journal of Pharmacy Practice, 5(2):49-52.

6. Bagcchi, S. (2014). Hypothyroidism in India: more to be done. Lancet Diabetes Endocrinol, 2(10):778.

7. Kangelaris, G. T., Kim, T. B., \& Orloff, L. A. (2010). Role of ultrasound in thyroid disorders. Otolaryngologic Clinics of North America, 43(6), 1209-1227.

8. Chaudhary, V., \& Bano, S. (2013). Thyroid ultrasound. Indian Journal of Endocrinology and Metabolism, 17(2):219-227.

9. Wong, K. T., Yolanda, Y. P., \& Lee. (2014). Thyroid gland. In: Ahuja, A. T., ed. Diagnostic ultrasound: head and neck. 1st edn. Amirsys Publishing, 52. 